\title{
Agreement between MRI and pathologic breast tumor size after neoadjuvant chemotherapy, and comparison with alternative tests: individual patient data meta-analysis
}

Michael L. Marinovich ${ }^{1 *}$, Petra Macaskill' ${ }^{1}$ Les Irwig ${ }^{1}$, Francesco Sardanelli², Eleftherios Mamounas ${ }^{3}$, Gunter von Minckwitz ${ }^{4}$, Valentina Guarneri ${ }^{5}$, Savannah C. Partridge ${ }^{6}$, Frances C. Wright ${ }^{7}$, Jae Hyuck Choi $^{8}$, Madhumita Bhattacharyya ${ }^{9}$, Laura Martincich ${ }^{10}$, Eren Yeh ${ }^{11}$, Viviana Londero ${ }^{12}$ and Nehmat Houssami ${ }^{1}$

\begin{abstract}
Background: Magnetic resonance imaging (MRI) may guide breast cancer surgery by measuring residual tumor size post-neoadjuvant chemotherapy (NAC). Accurate measurement may avoid overly radical surgery or reduce the need for repeat surgery. This individual patient data (IPD) meta-analysis examines MRI's agreement with pathology in measuring the longest tumor diameter and compares MRI with alternative tests.

Methods: A systematic review of MEDLINE, EMBASE, PREMEDLINE, Database of Abstracts of Reviews of Effects, Heath Technology Assessment, and Cochrane databases identified eligible studies. Primary study authors supplied IPD in a template format constructed a priori. Mean differences (MDs) between tests and pathology (i.e. systematic bias) were calculated and pooled by the inverse variance method; limits of agreement (LOA) were estimated. Test measurements of $0.0 \mathrm{~cm}$ in the presence of pathologic residual tumor, and measurements $>0.0 \mathrm{~cm}$ despite pathologic complete response (pCR) were described for MRI and alternative tests.
\end{abstract}

Results: Eight studies contributed IPD ( $N=300)$. The pooled MD for MRI was $0.0 \mathrm{~cm}(\mathrm{LOA}:+/-3.8 \mathrm{~cm})$. Ultrasound underestimated pathologic size $(M D:-0.3 \mathrm{~cm})$ relative to MRI $(M D: 0.1 \mathrm{~cm})$, with comparable LOA. MDs were similar for MRI $(0.1 \mathrm{~cm})$ and mammography $(0.0 \mathrm{~cm})$, with wider LOA for mammography. Clinical examination underestimated size (MD: $-0.8 \mathrm{~cm}$ ) relative to MRI (MD: $0.0 \mathrm{~cm}$ ), with wider LOA. Tumors "missed" by MRI typically measured $2.0 \mathrm{~cm}$ or less at pathology; tumors $>2.0 \mathrm{~cm}$ were more commonly "missed" by clinical examination (9.3\%). MRI measurements $>5.0 \mathrm{~cm}$ occurred in $5.3 \%$ of patients with pCR, but were more frequent for mammography (46.2\%).

Conclusions: There was no systematic bias in MRI tumor measurement, but LOA are large enough to be clinically important. MRI's performance was generally superior to ultrasound, mammography, and clinical examination, and it may be considered the most appropriate test in this setting. Test combinations should be explored in future studies.

Keywords: Breast cancer, Neoadjuvant chemotherapy, Magnetic resonance imaging, Tumor response, Monitoring

\footnotetext{
* Correspondence: luke.marinovich@sydney.edu.au

'Screening and Test Evaluation Program (STEP), Sydney School of Public Health, The University of Sydney, A27, Edward Ford Building, Sydney, NSW 2006, Australia

Full list of author information is available at the end of the article
}

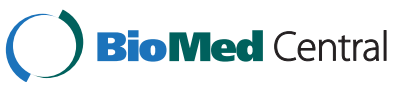

(c) 2015 Marinovich et al. Open Access This article is distributed under the terms of the Creative Commons Attribution 4.0 International License (http://creativecommons.org/licenses/by/4.0/), which permits unrestricted use, distribution, and reproduction in any medium, provided you give appropriate credit to the original author(s) and the source, provide a link to the Creative Commons license, and indicate if changes were made. The Creative Commons Public Domain Dedication waiver (http://creativecommons.org/publicdomain/zero/1.0/) applies to the data made available in this article, unless otherwise stated. 


\section{Background}

Magnetic resonance imaging (MRI) has been proposed to have a role in guiding breast cancer surgery by measuring the size of residual tumor after neoadjuvant chemotherapy (NAC), and has been shown to have high sensitivity for detecting residual disease [1]. Given that guidelines recommend assessment of the largest tumor diameter [2], estimation of the largest diameter by MRI may guide decisions about whether subsequent mastectomy or breast conserving surgery (BCS) should be attempted, as well as assist in planning resection to achieve clear margins in BCS. Underestimation of tumor size may therefore lead to involved surgical margins and repeat surgery; overestimation may lead to overly radical surgery (including mastectomy when BCS may have been possible), and poorer cosmetic and psychosocial outcomes [3].

Tumor size measurement is subject to potential errors, and both tumor characteristics and imaging limitations may differentially affect the measurement accuracy of tests used for this purpose. MRI may over- or underestimate tumor size due to artefacts such as partial volume effects [4] or disruptions to signal intensity from marker placement [5]. Tumors may not be well visualised by mammography in patients with dense breasts [6] or multifocal cancer [7]. Ultrasound (US) measurements may be compromised by unclear margins [8], acoustic shadowing [9] or limitations in the field of view [10]. Imaging modalities also differ in their ability to visualise ductal carcinoma in situ (DCIS) [11]. The inherent pliability of breast tissue also means that tumor dimensions may vary depending on patient positioning [12]; therefore, differences in measurements undertaken in upright (mammography), supine (US) and prone positions (MRI) may arise. Furthermore, the effects of NAC may introduce greater bias in residual tumor measurement relative to the preoperative setting: reactive inflammation, fibrosis or necrosis may be difficult to distinguish from residual tumor [13], and measurement errors may be additive when tumors regress as multiple, scattered deposits [2].

While many studies have sought to assess the relative ability of MRI and other tests to estimate tumor size after NAC, conclusions have been hampered by small sample sizes and inadequate statistical methods. A previous study-level meta-analysis demonstrated that misleading conclusions about the accuracy of MRI may result from inappropriate analytic methods that do not measure agreement between clinical measures (e.g. Pearson or Spearman correlation coefficients) [14]. However, that meta-analysis was limited in its ability to estimate the agreement between MRI and pathologic measurements, and to compare MRI with alternative tests, due to numerous shortcomings in the available data. For example, inconsistencies in measurement between studies, such as the inclusion or exclusion of residual ductal carcinoma in situ (DCIS) in pathologic tumour measurements, may differentially affect the measurement accuracy of MRI and other tests, and also limit the clinical applicability of pooled estimates. Comparison of MRI and other tests was also hampered by the tests being reported for different (or, at best, overlapping) patient groups, for which test performance may vary. Furthermore, a fundamental limitation was that assessing the validity of assumptions underlying the recommended statistical methods (mean differences and limits of agreement [15]) was often not possible due to inadequate reporting.

To address those limitations, we investigated agreement between MRI-measured and pathologic tumor size after NAC in an individual patient data (IPD) metaanalysis of a large number of breast cancer patients, using appropriate methods for evaluating the agreement between measurements [15]. Key differences between this and the previous study-level meta-analysis are summarised in Additional file 1: Appendix 1. The IPD methodology allowed us to standardise tumor measurements to include invasive cancer only, explore agreement only when residual tumor is truly present, and describe MRI measurement errors in detail. In addition, our study extended previous work by exploring agreement by characteristics that have been suggested to contribute to inaccurate measurement (NAC agents and HER2 status) [16, 17], and examining MRI's agreement compared with and in addition to alternative tests (US, mammography, clinical examination) when the tests were conducted in the same patients [18].

\section{Methods}

\section{Identification of studies}

A systematic literature search up to February 2011 was undertaken to identify studies of MRI for measuring residual tumor after NAC. MEDLINE and EMBASE were searched via EMBASE.com; PREMEDLINE, Database of Abstracts of Reviews of Effects, Heath Technology Assessment, and Cochrane databases were searched via Ovid. Search terms linked MRI with breast cancer and response to NAC. Keywords and medical subject headings included 'breast cancer', 'nuclear magnetic resonance imaging', 'MRI', 'neoadjuvant', and 'response'. The full search strategy has been reported previously $[1,19]$. Reference lists were also searched and content experts consulted to identify additional studies.

\section{Review of studies and eligibility criteria}

Abstracts were screened for eligibility by one author (MLM); a sample of $10 \%$ was assessed independently $(\mathrm{NH})$ to ensure consistent application of eligibility criteria. There were no changes to eligibility criteria or coding schemes based on the independent assessment. Eligible studies enrolled $\geq 15$ patients with newly diagnosed breast 
cancer undergoing NAC, with MRI and at least one other test (US, mammography, clinical examination) after NAC to assess residual tumor size (longest diameter) prior to surgery.

Potentially eligible citations were reviewed in full (MLM or NH). The screening and inclusion process is summarised in Additional file 1: Appendix 2.

\section{Individual patient data}

A research protocol and database template were drafted $a$ priori, specifying the study rationale and objectives, IPD requirements, and planned statistical analyses (Additional file 1: Appendix 3). Those documents were forwarded to the authors of eligible studies with an invitation to participate in the IPD meta-analysis, with email follow-up if no response was received.

For each participating study, data irregularities were discussed with the authors. Non-numeric tumor measurements were treated as missing data. Observations with missing pathologic measurements were excluded. Pathologic measurements considered residual invasive components only; therefore, the definition of pathologic complete response (pCR) was standardised across studies as the absence of residual invasive cancer, with or without the presence of DCIS (i.e. a pathologic measurement of $0.0 \mathrm{~cm}$ ) [20].

\section{Statistical analysis}

For individual studies, Bland-Altman scatterplots of the differences between measurements by the relevant tests and pathology (vertical axis) and their mean (horizontal axis) were constructed. Plots were examined to assess whether the differences were normally distributed and independent from the underlying size of the measurements [15]. Scatterplots of log-transformed measurements were also constructed to assess whether underlying relationships were improved. Preliminary mixed linear models (PROC MIXED in SAS) of the difference between measurements by their mean, and pathologic size by MRI size, were unstable and are not reported.

For patients with residual tumor at pathology, measurement biases were estimated as the absolute mean differences (MDs) between MRI, comparator tests and pathology; the associated $95 \%$ limits of agreement (LOA) were also calculated for each study [15]. Relative MDs were derived by exponentiation of the difference of logtransformed measurements. MDs were pooled by the inverse variance method using RevMan 5.2. A fixed effect was assumed unless statistically significant heterogeneity was present, as assessed by the Cochrane Q statistic. The extent of heterogeneity was quantified by the $\mathrm{I}^{2}$ statistic [21]. To estimate the $95 \%$ LOA for a pooled MD, a pooled variance was computed under the assumption that the variance of the differences was equal across studies.
The pooled variance was calculated as the weighted average of these within-study variances, weighted by the corresponding degrees of freedom for each study (i.e. an extension of the approach used for a two sample $t$-test [22]).

In addition, test measurements of $0.0 \mathrm{~cm}$ in the presence of pathologic residual tumor, and measurements $>0.0 \mathrm{~cm}$ despite pCR were described for MRI and comparator tests. Exact $95 \%$ confidence intervals for proportions were computed (SAS version 9.2). Paired differences between tests were tested with McNemar's test. Differences in characteristics between patients with and without tumor measurements by comparator tests were compared with independent samples t-tests for continuous variables and with chi-squared or Fisher's exact tests for categorical variables.

All tests of statistical significance were two-sided. Except for tests of heterogeneity $(p<0.10)$, the level chosen for statistical significance was $p<0.05 ; p \leq 0.10$ was considered to represent weak evidence of a difference [23].

\section{Results \\ Study characteristics}

A total of 2108 citations were identified. Twenty-four studies (1228 patients) were eligible for inclusion [13, 24-46]; eight of those contributed IPD to this analysis (300 patients) $[13,24,25,29,34,38,44,46]$ (Additional file 1: Appendix 2). Agreement between residual tumor size by tests and pathology was compared for MRI and US in five studies [13, 29, 34, 38, 46]; MRI and mammography in four studies [13, 24, 34, 38]; and MRI and clinical examination in three studies [13, 24, 25]. For one study [44], MRI and pathologic measurements were provided but data for alternative tests were unavailable.

Characteristics of the included studies are presented in Table 1. Included studies were generally representative of the broader population of studies reported previously, based qualitative comparison of aggregate descriptive characteristics [14]. However, patients in this analysis were more likely to have had T3 tumors or stage III disease; were more commonly treated with anthracycline-taxane-based NAC; and had a shorter time between MRI and surgery.

Technical characteristics of MRI are presented in Additional file 1: Appendix 4. The majority of studies used dynamic contrast-enhanced MRI ( $88 \%$ ) with a 1.5 -T magnet $(75 \%)$. Dedicated bilateral breast coils were used in all studies reporting the coil type. All studies providing detail on contrast employed gadolinium-based materials, most commonly gadopentetate dimeglumine (62\%), at the standard dosage of $0.1 \mathrm{mmol} / \mathrm{kg}$ body weight $(75 \%)$.

Pathology from surgical excision was the reference standard for all patients in all but one study [34], where pCR was verified by localisation biopsy in two cases ( $0.7 \%$ of all patients). 
Table 1 Summary of cohort, tumour, treatment and reference standard characteristics of studies included in the individual patient data analysis

\begin{tabular}{|c|c|c|c|c|}
\hline \multirow[b]{2}{*}{ Variable } & \multirow[b]{2}{*}{ Patients (\%) } & \multicolumn{3}{|c|}{ Study level estimates } \\
\hline & & Median & IQR & Range \\
\hline \multicolumn{5}{|l|}{ Cohort characteristics } \\
\hline N patients with MRI (8 studies) & $300(N A)$ & 36 & $28-50$ & $13-59$ \\
\hline Recruitment mid-point (year) (4 studies) & $144(\mathrm{NA})$ & 2003 & $2001-2005$ & $2001-2006$ \\
\hline Age, mean or median (years) (8 studies) & 300 (NA) & 47 & $46-48$ & $43-49$ \\
\hline \multicolumn{5}{|l|}{ Menopausal status (\%) (2 studies) } \\
\hline Pre & $51(72.9)$ & 64.6 & $60.4-68.8$ & $60.4-68.8$ \\
\hline Peri/post & $19(27.1)$ & 25.0 & $18.8-31.2$ & $18.8-31.2$ \\
\hline \multicolumn{5}{|l|}{ Tumour characteristics } \\
\hline Clinical size, mean or median $(\mathrm{cm})^{\mathrm{a}}$ (4 studies) & $136(N A)$ & 4.6 & $4.2-6.6$ & $4.0-8.2$ \\
\hline \multicolumn{5}{|l|}{ T stage (\%) ${ }^{\mathrm{a}}$ (4 studies) } \\
\hline $\mathrm{T} 1$ & $5(2.9)$ & 2.9 & $1.0-5.0$ & $0.0-6.2$ \\
\hline $\mathrm{T} 2$ & $62(35.8)$ & 43.0 & $23.9-50.3$ & $6.2-56.2$ \\
\hline T3 & $78(45.1)$ & 43.6 & $38.3-49.1$ & $37.5-50.0$ \\
\hline T4 & $28(16.2)$ & 9.8 & $0.0-30.6$ & $0.0-41.7$ \\
\hline \multicolumn{5}{|l|}{ Stage (\%) (6 studies) } \\
\hline 1 & $1(0.5)$ & 0.0 & $0.0-0.0$ & $0.0-3.1$ \\
\hline$\|$ & $131(59.0)$ & 66.1 & $45.0-78.0$ & $27.1-86.7$ \\
\hline III & $83(37.4)$ & 32.3 & $22.0-37.5$ & $0.0-72.9$ \\
\hline IV & $7(3.2)$ & 0.0 & $0.0-0.0$ & $0.0-17.5$ \\
\hline \multicolumn{5}{|l|}{ Histology (\%) (6 studies) } \\
\hline IDC & $191(84.1)$ & 86.2 & $74.2-87.8$ & $68.8-90.0$ \\
\hline ILC or IDC/ILC & $19(8.4)$ & 9.8 & $5.1-10.0$ & $4.9-18.8$ \\
\hline Other & $17(7.5)$ & 7.9 & $4.0-12.5$ & $0.0-16.1$ \\
\hline \multicolumn{5}{|l|}{ Nodal status (\%) (4 studies) } \\
\hline Positive & $109(72.2)$ & 71.0 & $62.5-80.6$ & $56.2-87.8$ \\
\hline Negative & $42(27.8)$ & 29.0 & $19.4-37.5$ & $12.2-43.8$ \\
\hline \multicolumn{5}{|l|}{ ER $(\%)^{a}$ (5 studies) } \\
\hline Positive & $113(60.1)$ & 62.5 & $60.0-64.4$ & $45.0-69.2$ \\
\hline Negative & 73 (38.8) & 37.5 & $32.2-40.0$ & $15.4-55.0$ \\
\hline Unknown or NR & $2(1.1)$ & 0.0 & $0.0-0.0$ & $0.0-3.4$ \\
\hline \multicolumn{5}{|l|}{ PR (\%) (4 studies) } \\
\hline Positive & $71(44.9)$ & 41.2 & $32.9-49.8$ & $30.8-52.1$ \\
\hline Negative & $84(53.2)$ & 51.5 & $47.5-59.4$ & $48.5-65.0$ \\
\hline Unknown or NR & $3(1.9)$ & 1.0 & $0.0-2.7$ & $0.0-3.4$ \\
\hline \multicolumn{5}{|l|}{ HER2 (\%) (3 studies) } \\
\hline Positive & $42(28.8)$ & 29.2 & $22.5-33.9$ & $22.5-33.9$ \\
\hline Negative & $97(66.4)$ & 62.5 & $61.0-77.5$ & $61.0-77.5$ \\
\hline Unknown or NR & $7(4.8)$ & 5.1 & $0.0-8.3$ & $0.0-8.3$ \\
\hline
\end{tabular}


Table 1 Summary of cohort, tumour, treatment and reference standard characteristics of studies included in the individual patient data analysis (Continued)

\begin{tabular}{|c|c|c|c|c|}
\hline Treatment & & & & \\
\hline NAC regimen $(\%)^{a}$ (8 studies) & & & & \\
\hline Anthracycline-based & $115(38.1)$ & 9.3 & $0.0-77.6$ & $0.0-100.0$ \\
\hline Antracycline-taxane-based & $181(59.9)$ & 88.1 & $17.4-100.0$ & $0.0-100.0$ \\
\hline Other & $6(2.0)$ & 0.0 & $0.0-2.5$ & $0.0-100.0$ \\
\hline Trastuzumab (\%) (3 studies) & & & & \\
\hline Trastuzumab used & 29 (19.6) & 7.3 & $2.1-42.4$ & $2.1-42.4$ \\
\hline Trastuzumab not used & $119(80.4)$ & 92.7 & $57.6-97.9$ & $57.6-97.9$ \\
\hline Type of surgery (\%) (8 studies) & & & & \\
\hline BCS & $132(43.1)$ & 50.3 & $37.6-55.9$ & $6.2-59.4$ \\
\hline Mastectomy & $172(56.2)$ & 57.2 & $44.1-63.8$ & $34.4-93.8$ \\
\hline No surgery & $2^{b}(0.7)$ & 0.0 & $0.0-0.0$ & $0.0-6.2$ \\
\hline Reference standard & & & & \\
\hline Type of reference standard (\%) (8 studies) & & & & \\
\hline Pathology & $298(99.3)$ & 100.0 & $100.0-100.0$ & $93.8-100.0$ \\
\hline Other & $2^{b}(0.7)$ & 0.0 & $0.0-0.0$ & $0.0-6.2$ \\
\hline Time from MRI to surgery, mean or median/estimate (days) (6 studies) & $228(\mathrm{NA})$ & 16 & $12-25$ & $7-28$ \\
\hline Prevalence of pCR (\%) (8 studies) & $300(\mathrm{NA})$ & 19.0 & $15.5-23.4$ & $7.1-27.5$ \\
\hline
\end{tabular}

$B C S$ breast conserving surgery, $D C I S$ ductal carcinoma in situ, ER estrogen receptor, HER2 human epidermal growth factor receptor 2, IDC invasive ductal carcinoma, ILC invasive lobular carcinoma, IQR inter-quartile range, MRI magnetic resonance imaging, NA not applicable, NAC neoadjuvant chemotherapy, NR not reported, $p C R$ pathologic complete response, $P R$ progesterone receptor

${ }^{a}$ Calculation of values based on total number of patients enrolled, a minority of whom may not have contributed data to this analysis

bocalisation biopsy showed the absence of residual tumour (i.e. pathologic measurement of $0.0 \mathrm{~cm}$ )

\section{MRI when residual tumor present at pathology}

Figure 1a describes the size of residual tumor present at pathology $(N=243)$ that was "missed" by MRI (i.e. MRI tumor measurements of $0.0 \mathrm{~cm}$ ). Patients for whom MRI truly detected residual tumor (i.e. measurements $>0.0 \mathrm{~cm}$ ) are also included in the column labelled "not applicable" (N/A). Pathologic measurements of tumors "missed" by MRI ranged between $0.1-11.0 \mathrm{~cm}$ (median $=0.6 \mathrm{~cm}$ ), and measured 0.1-1.0 cm for 12 patients (4.9\%); 1.1-2.0 cm for four patients (1.6\%); 2.1-3.0 cm for one patient $(0.8 \%)$; and $>7.0 \mathrm{~cm}$ for one patient $(0.8 \%)$.

Study-specific Bland-Altman plots, MDs and LOA between MRI and pathology are presented in Additional file 1: Appendix 5. The plots suggested a tendency in some studies for larger differences with increasing tumor size; underlying relationships were not uniformly improved by log transformation (Additional file 1: Appendix 5). Similar relationships were also apparent for US, mammography and clinical examination (Additional file 1: Appendices 6-8). Analyses of absolute differences between tests and pathology are reported here; analyses of relative (log) differences were comparable, and are presented in Additional file 1: Appendices 9-10.

Meta-analysis of MDs between MRI and pathology (Table 2; Additional file 1: Appendix 11) showed no systematic bias in MRI's estimation of pathologic tumor size (pooled $\mathrm{MD}=0.0 \mathrm{~cm}$ [95 \% CI: $-0.1-0.2 \mathrm{~cm}]$ ), with no evidence of heterogeneity $\left(\mathrm{I}^{2}=0 \%\right)$. Scatterplots showed both over- and underestimation by MRI (Additional file 1: Appendix 5). Pooled LOA indicated that $95 \%$ of pathologic measurements fall between $+/-3.8 \mathrm{~cm}$ of the MRI measurement.

\section{MRI versus US}

In 123 patients with pathologic residual tumor and paired measurements by MRI and US, distributions of pathologic size were comparable when either test measured $0.0 \mathrm{~cm}$; tumors "missed" by each test typically measured $\leq 2.0 \mathrm{~cm}$, with one MRI measurement in the range of $2.1-3.0 \mathrm{~cm}$ (Fig. 1b).

Pooled MDs showed a tendency for MRI to slightly overestimate pathologic tumor size $(\mathrm{MD}=0.1 \mathrm{~cm})$ with no evidence of heterogeneity $\left(\mathrm{I}^{2}=0 \%\right.$ ) (Table 2; Additional file 1: Appendix 11). A larger tendency for underestimation by US $(\mathrm{MD}=-0.3 \mathrm{~cm})$ was observed with substantial heterogeneity $\left(\mathrm{Q}=13.11, \mathrm{df}=4, p=0.01 ; \mathrm{I}^{2}=69 \%\right)$; the pooled $\mathrm{MD}$ did not change when a fixed or random effect(s) were assumed. Pooled differences between MRI and US showed only weak evidence of a difference between the measurements (assuming random effects, $p=0.10$ ). Pooled LOA were comparable for MRI $(+/-2.8 \mathrm{~cm})$ and US $(+/-2.6 \mathrm{~cm})$ (Table 2), with both over- and underestimation observed 
(a) MRI alone $(\mathrm{N}=243)$

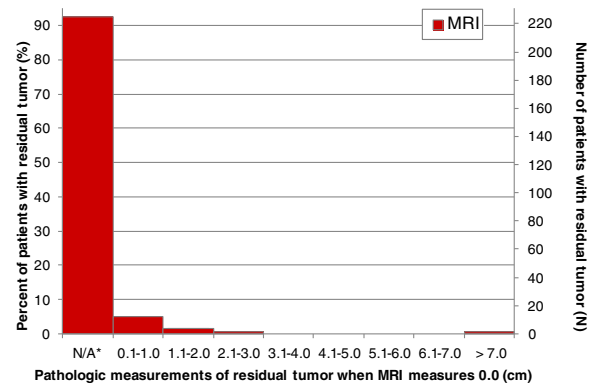

(c) MRI versus mammography $(\mathrm{N}=78)$

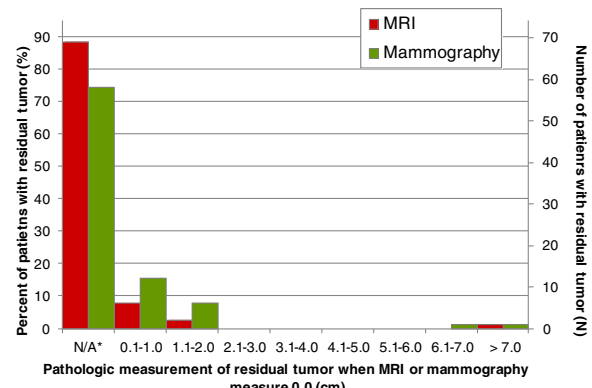

(b) MRI versus US $(\mathrm{N}=123)$

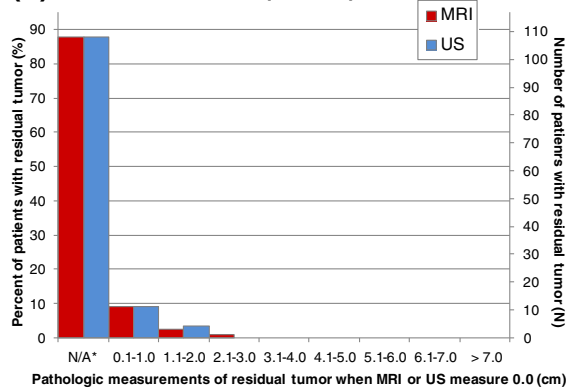

(d) MRI versus clinical examination ( $\mathrm{N}=107)$

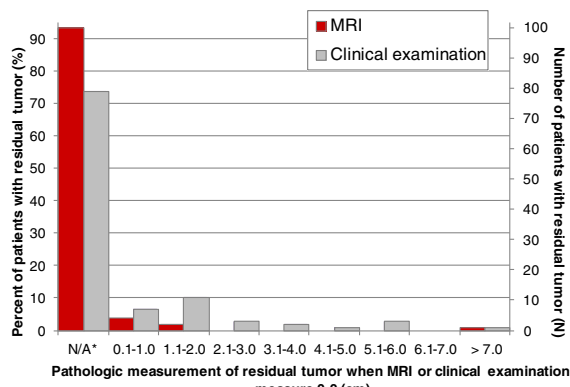

Fig. 1 Pathologic size $(\mathrm{cm})$ of tumor "missed" by MRI for: a all patients with residual tumor $(N=243)$; and compared with $\mathbf{b}$ US ( $N=123)$, c mammography $(N=78)$, and $\mathbf{d}$ clinical examination $(N=107)$. MRI = magnetic resonance imaging; $N / A=$ not applicable; US = ultrasound. *Pathology and test(s) measure $>0.0 \mathrm{~cm}$ (i.e. residual tumor was not "missed" by MRI or alternative tests).

for both tests (Additional file 1: Appendices 5-6). Combining MRI and US measurements by taking their mean resulted in slight underestimation $(\mathrm{MD}=-0.1 \mathrm{~cm})$, with a small reduction in LOA compared with either test alone $(+/-2.3 \mathrm{~cm})$.

US measurements were not possible (due to large or diffuse lesions, or acoustic shadowing on US images) in 14 patients (10.2 \% of patients with MRI). Patients without US had significantly larger tumors at pathology (mean 5.3 vs $2.0 \mathrm{~cm} ; p=0.003$ ); were more likely to be diagnosed with advanced (stage III/IV) disease (83.3\% vs $32.3 \% ; p=0.001$ ); were less likely to have received taxane-based NAC (38.5\% vs $74.0 \% ; p=0.02$ ); and were more likely to have undergone mastectomy (78.6 \% vs $46.3 \% ; p=0.02$ ) than patients with US measurements. For the 14 patients without US, the MD between MRI and pathology was $-1.5 \mathrm{~cm}(95 \% \mathrm{CI}:-3.1-0.1 \mathrm{~cm})$ and the LOA were $+/-6.0 \mathrm{~cm}$ (Table 2).

\section{MRI versus mammography}

For patients with pathologic residual tumor and measurements by MRI and mammography $(N=78)$, tumors with measurements of $0.0 \mathrm{~cm}$ by the tests typically measured $\leq 2.0 \mathrm{~cm}$ at pathology (Fig. 1c); however, the proportion of "missed" tumors within that range was higher for mammography (23.1 \%) than MRI (10.3 \%; $p=0.002)$. Mammography "missed" two tumors measuring $>6.0 \mathrm{~cm}$; one of those (measuring $11.0 \mathrm{~cm}$ ) also measured $0.0 \mathrm{~cm}$ on MRI.

Pooled MDs showed a tendency for MRI to slightly overestimate pathologic tumor size $(\mathrm{MD}=0.1 \mathrm{~cm})$ with no evidence of heterogeneity $\left(\mathrm{I}^{2}=0 \%\right.$ ) (Table 2; Additional file 1 : Appendix 11). No systematic bias was observed for mammography $(\mathrm{MD}=0.0 \mathrm{~cm})$, but moderate heterogeneity was present $\left(\mathrm{I}^{2}=39 \%\right)$. No evidence of a difference between MRI and mammographic measurements was observed (assuming a fixed effect, $p=0.59$ ). Pooled LOA for mammography $(+/-5.0 \mathrm{~cm})$ were wider than for MRI (+/-4.1 cm) (Table 2); over- and underestimation were observed for both tests (Additional file 1: Appendices 5 and 7). Combining MRI and mammography by taking their mean did not improve the MD $(0.1 \mathrm{~cm})$ or LOA $(+/-4.2 \mathrm{~cm})$ over MRI alone.

Tumor measurements by mammography were not possible (due to dense breasts, tumor margins no longer being assessable, or tumor not being visible) for 25 patients $(24.3 \%$ of patients with MRI). Patients without mammography were significantly younger (mean 42 vs 47 years; $p=0.03$ ) than patients with mammographic measurements. For those patients, the MD between MRI and pathology was $0.0 \mathrm{~cm}(95 \% \mathrm{CI}-0.7-0.7 \mathrm{~cm})$ and the LOA were $+/-3.5 \mathrm{~cm}$ (Table 2). 
Table 2 Pooled absolute differences ( $\mathrm{cm}$ ) (fixed effect unless noted) and limits of agreement for studies and patients comparing the respective tests

\begin{tabular}{|c|c|c|c|c|c|}
\hline & $\mathrm{N}$ (studies) & $\mathrm{N}$ (patients) & Pooled MD (cm) $(95 \% \mathrm{Cl})$ & $1^{2}$ & $\operatorname{LOA}(\mathrm{cm})$ \\
\hline \multicolumn{6}{|l|}{ All studies and patients } \\
\hline MRI vs pathology & 8 & 243 & $0.0(-0.1,0.2)$ & $0 \%$ & $+/-3.8$ \\
\hline \multicolumn{6}{|l|}{ Studies of MRI vs US } \\
\hline MRI vs pathology & 5 & 123 & $0.1(-0.2,0.3)$ & $0 \%$ & $+/-2.8$ \\
\hline US vs pathology ${ }^{a}$ & 5 & 123 & $-0.3(-0.6,0.1)$ & $69 \%$ & $+/-2.6$ \\
\hline MRI and US (mean) vs pathology & 5 & 123 & $-0.1(-0.3,0.1)$ & $16 \%$ & $+/-2.3$ \\
\hline MRI vs US ${ }^{a}$ & 5 & 123 & $0.3(-0.1,0.7)$ & $81 \%$ & NA \\
\hline MRI vs pathology (patients without US) & 3 & 14 & $-1.5(-3.1,0.1)$ & NA & $+/-6.0$ \\
\hline \multicolumn{6}{|l|}{ Studies of MRI vs mammography } \\
\hline MRI vs pathology & 4 & 78 & $0.1(-0.1,0.3)$ & $0 \%$ & $+/-4.1$ \\
\hline Mammography vs pathology & 4 & 78 & $0.0(-0.3,0.4)$ & $39 \%$ & $+/-5.0$ \\
\hline MRI and mammography (mean) vs pathology & 4 & 78 & $0.1(-0.1,0.4)$ & $21 \%$ & $+/-4.2$ \\
\hline MRI vs mammography & 4 & 78 & $0.1(-0.2,0.4)$ & $0 \%$ & NA \\
\hline MRI vs pathology (patients without mammography) & 3 & 25 & $0.0(-0.7,0.7)$ & NA & $+/-3.5$ \\
\hline \multicolumn{6}{|l|}{ Studies of MRI vs clinical examination } \\
\hline MRI vs pathology & 3 & 107 & $0.0(-0.2,0.3)$ & $0 \%$ & $+/-4.2$ \\
\hline Clinical examination vs pathology ${ }^{a}$ & 3 & 107 & $-0.8(-1.5,-0.1)^{*}$ & $57 \%$ & $+/-5.1$ \\
\hline MRI and clinical examination (mean) vs pathology & 3 & 107 & $-0.2(-0.5,0.1)$ & $9 \%$ & $+/-4.1$ \\
\hline MRI vs clinical examination ${ }^{a}$ & 3 & 107 & $0.9(0.2,1.5)^{*}$ & $56 \%$ & NA \\
\hline MRI vs pathology (patients without clinical examination) ${ }^{b}$ & 2 & 3 & $N A^{c}$ & $N A^{c}$ & $N A^{c}$ \\
\hline
\end{tabular}

$\mathrm{Cl}$ confidence interval, $L O A$ limits of agreement, MD mean difference, MRI magnetic resonance imaging, NA not applicable, US ultrasound ${ }^{*} p<0.01$

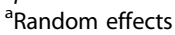

${ }^{b}$ Patients without comparator test combined as a single data set. Pooled meta-analysis not undertaken

${ }^{c}$ Not calculated due to small number of patients

\section{MRI versus clinical examination}

For 107 patients with pathologic residual tumor and paired measurements by MRI and clinical examination, tumors "missed" by MRI measured $\leq 2.0 \mathrm{~cm}$ at pathology in all but one case $(0.9 \%)$, but 10 patients $(9.3 \%)$ with measurements of $0.0 \mathrm{~cm}$ by clinical examination had pathologic residual tumor $>2.0 \mathrm{~cm}(p=0.003)$. Both tests "missed" one tumor with a pathologic measurement of $11.0 \mathrm{~cm}$ (Fig. 1d).

Pooled MDs showed no systematic bias in MRI's estimation of pathologic tumor size $(\mathrm{MD}=0.0 \mathrm{~cm})$ with no evidence of heterogeneity $\left(\mathrm{I}^{2}=0 \%\right.$ ) (Table 2; Additional file 1: Appendix 11). A relatively large tendency for underestimation by clinical examination $(\mathrm{MD}=-0.8 \mathrm{~cm})$ was observed with moderate heterogeneity $(\mathrm{Q}=4.65, \mathrm{df}=2$, $\left.p=0.1 ; \mathrm{I}^{2}=57 \%\right)$; the pooled MD assuming a fixed effect was similar $(\mathrm{MD}=-0.7 \mathrm{~cm})$. Pooled differences between MRI and clinical examination showed measurements by clinical examination to be significantly lower than MRI (assuming random effects, $p=0.006$ ). Pooled LOA for clinical examination $(+/-5.1 \mathrm{~cm})$ were wider than for MRI $(+/-4.2 \mathrm{~cm})$ (Table 2$)$; over- and underestimation were observed for both tests (Additional file 1: Appendices 5 and
8). Combining MRI and clinical examination by taking their mean did not substantially improve the MD $(-0.2 \mathrm{~cm})$ or LOA $(+/-4.1)$ over MRI alone.

Estimation of tumor size by clinical examination was not possible for three patients. In one patient each, MRI correctly estimated, underestimated $(-0.1 \mathrm{~cm})$ and overestimated $(0.8 \mathrm{~cm})$ pathologic tumor size.

\section{MRI measurement by NAC agents and HER2 status}

In 88 patients treated with non-taxane-based NAC from three studies $[25,29,46]$, the pooled MD showed slight underestimation by MRI $(-0.1 \mathrm{~cm})$ with no evidence of heterogeneity $\left(\mathrm{I}^{2}=0 \%\right)$. Data from 63 patients treated with taxane-containing NAC in those studies showed a tendency for overestimation by MRI $(\mathrm{MD}=0.2 \mathrm{~cm})$ with no evidence of heterogeneity $\left(\mathrm{I}^{2}=0 \%\right)$ (Additional file 1 : Appendix 12). Pooled LOA in patients treated with nontaxane-based NAC $(+/-4.3 \mathrm{~cm})$ were wider than for patients treated with taxanes $(+/-2.8 \mathrm{~cm})$. When three additional studies $[13,24,38]$ using only taxane-containing NAC were included in pooled estimates (six studies, 152 patients in total), the MD did not change $(0.2 \mathrm{~cm}$; $\left.\mathrm{I}^{2}=0 \%\right)$, but LOA were higher $(+/-3.9 \mathrm{~cm})$. 
Pooled MDs from three studies $[24,29,46]$ showed comparable overestimation by MRI in HER2- $(\mathrm{MD}=0.2 \mathrm{~cm}$; $N=97)$ and HER2+ patients (MD $=0.3 \mathrm{~cm} ; N=42)$, with no evidence of heterogeneity for either group $\left(\mathrm{I}^{2}=0 \%\right)$ (Additional file 1: Appendix 12). Pooled LOA were also similar (+/-4.3 cm for HER2- patients; $+/-4.2 \mathrm{~cm}$ for HER2+ patients).

\section{MRI when no residual tumor at pathology ( $\mathrm{pCR}$ )}

For all studies combined, pCR was present in 57/300 patients (19.0\% [95 \% CI: 14.7-23.9 \%]). Study-specific rates of pCR ranged from 7.1-27.5 \% (median $=19.1 \%$ ). MRI tumor measurements $>0.0 \mathrm{~cm}$ for patients with pCR are presented in Fig. 2a (measurements of $0.0 \mathrm{~cm}$ are also described, representing true identification of pCR by MRI). MRI measurements $>0.0 \mathrm{~cm}$ ranged between 0.3-6.1 cm (median $=2.0 \mathrm{~cm}$ ), and measured $0.1-1.0 \mathrm{~cm}$ for seven patients $(12.3 \%)$; $1.1-2.0 \mathrm{~cm}$ for six patients (10.5\%); $2.1-5.0 \mathrm{~cm}$ for five patients $(8.8 \%)$; and $>5.0 \mathrm{~cm}$ for three patients $(5.3 \%)$.

\section{$M R I$ versus alternative tests in assessing $p C R$}

Figure $2 \mathrm{~b}-\mathrm{d}$ present the distribution of MRI tumor measurements $>0.0 \mathrm{~cm}$ for patients with pCR compared with measurements by US $(N=35)$, mammography $(N=13$, excluding five patients with MRI but no mammographic measurement), and clinical examination $(N=18)$. Large $(>5.0 \mathrm{~cm})$ measurement errors in the presence of $\mathrm{pCR}$ were more common by mammography (46.2 \%) than MRI (15.4 \%; $p=0.05)$; both large MRI measurements also measured $>5.0 \mathrm{~cm}$ on mammography. The proportion of large MRI measurement errors was not significantly different from US or clinical examination.

For $5 / 18$ patients $(27.8 \%)$ with no mammographic measurement (due to dense breasts or tumor margins not being assessable post-NAC), MRI measurements $>0.0 \mathrm{~cm}$ occurred in three patients, ranging between $1.1-2.0 \mathrm{~cm}$.

\section{Discussion}

In the neoadjuvant setting, accurate measurement of residual malignancy may assist in guiding surgical management of breast cancer. While past research focussed on the accuracy of MRI to detect the absence of residual tumor (pCR) as a predictor of overall and disease-free survival [1], MRI measurements of tumor size have the potential to inform decisions about surgical extent (e.g. BCS versus mastectomy). Our IPD meta-analysis assessed the agreement between MRI and pathologic tumor measurements after NAC. Pooled MDs between MRI and pathology indicated that there was no systematic bias in MRI's (a) MRI alone ( $\mathrm{N}$ with $\mathrm{pCR}=57$ )

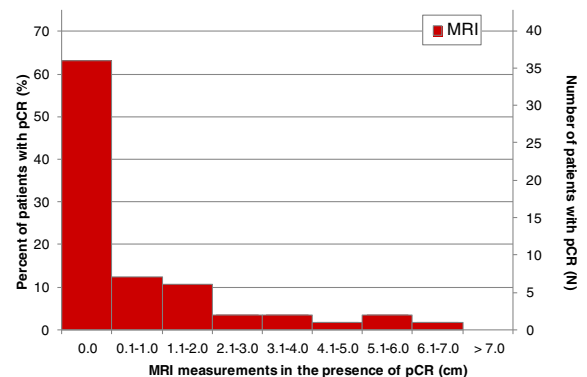

(c) MRI versus mammography ( $\mathrm{N}$ with $\mathrm{pCR}=13$ )

\section{(b) MRI versus US ( $\mathrm{N}$ with $\mathrm{pCR}=35$ )}

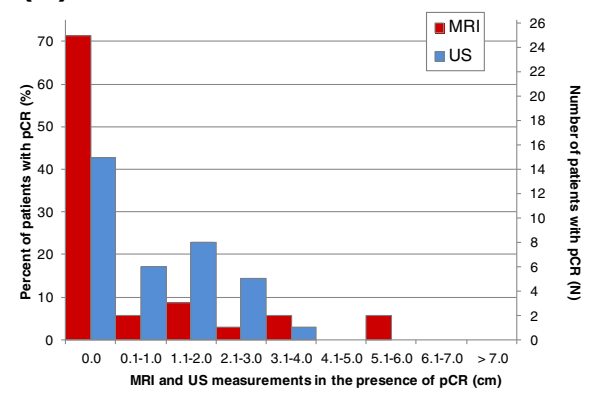

(d) MRI versus clinical exam ( $\mathrm{N}$ with $\mathrm{pCR}=18$ )
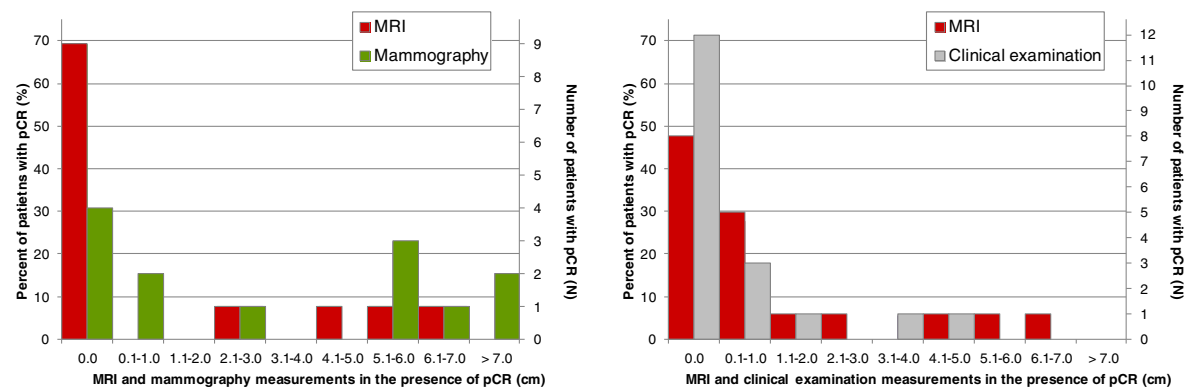

Fig. $2 \mathrm{MRI}$ measurements ( $\mathrm{cm})$ for: a all patients with $\mathrm{pCR}(\mathrm{N}=57)$; and compared with measurements by $\mathbf{b}$ US ( $N=35)$, c mammography $(\mathrm{N}=13)$, and $\mathbf{d}$ clinical examination $(N=18)$. Measurements of $0.0 \mathrm{~cm}$ denote correct identification of $\mathrm{pCR}$. MRI = magnetic resonance imaging; $\mathrm{pCR}=$ pathologic complete response; US = ultrasound 
estimation of tumor size when residual tumor was present. Measurement variability for agreement was lower than estimated by our previous study-level analysis [14]; however, both over- and underestimation by MRI were observed, and LOA $(+/-3.8 \mathrm{~cm})$ show that substantial disagreement with pathology is possible. MRI measurement errors within that range may be of clinical importance in terms of their implications for the choice of treatment.

The IPD methodology used in this analysis allowed for measurement errors to be explored in greater detail than that permitted by study-level analyses [14]. Tumors “missed' by MRI generally measured $\leq 2.0 \mathrm{~cm}$ at pathology; however, MRI measurements $>5.0 \mathrm{~cm}$ occurred in a small proportion of cases where pCR was achieved. Although descriptive reporting of such overestimation was not standard across included studies, one of the three cases of MRI measurements $>5 \mathrm{~cm}$ in the presence of pCR observed in this data set was attributed to the presence of extensive DCIS. Other possible causes include reactive inflammation, fibrosis or necrosis induced by NAC [13]. Description of cases of large overestimation in future studies would be valuable in guiding future research and practice. Assuming that surgeons consider the MRI-determined measurement when planning resection, such overestimation would lead to unnecessarily large excision. Although those patients are likely to benefit from improved disease-free and overall survival conferred by pCR [47], they are less likely to benefit from a reduction in surgical extent after NAC.

Comparisons of MRI and US in the same patients showed similar LOA, suggesting comparable performance by MRI and US when residual tumor is present (although substantial heterogeneity for US reflects its operator dependence [2]). However, contrary to our previous studylevel analysis [14], a small bias towards underestimation of tumor size was found for US; clinical preference for either slight overestimation (MRI) or underestimation (US) of pathologic size should be considered in the choice of test. Furthermore, our analysis extends previous work by suggesting that considering the mean measurement of both tests may further improve tumor measurement. Given that studies may not have interpreted MRI blinded to US, this result is likely to underestimate the value of combining the tests. Clinicians adopting this testing strategy should be aware that the direction of MRI's systematic bias was reversed (slight underestimation) when the tests were combined.

It is noteworthy that MRI did not estimate tumor size as accurately in patients for whom US measurement was not possible, with (on average) relatively large underestimation and wide LOA. Tumor characteristics are likely to have contributed to measurement being challenging for both tests. Patients without US had larger tumors (and consistent with this, were diagnosed with more advanced disease and were more likely to have undergone mastectomy), reflecting limitations in the US field of view [10]. The higher rate of non-taxane-based NAC in that group may also have contributed to the larger residual tumor size [48]. When planning resection, clinicians should note that although tumor measurement by MRI may be possible for such patients, the potential for size underestimation may lead to incomplete excision. This analysis is the first to consider those patients separately, and directly compare MRI and US when measurement by both tests can be undertaken. Our findings highlight the importance of study authors reporting MRI's agreement with pathology separately for patients with and without alternative tests $[14,18]$.

In patients with measurements by both MRI and mammography, a systematic bias in estimating tumor size was found only for MRI (slight overestimation); the larger overestimation for mammography found in a previous analysis (which included fewer studies comparing mammography and MRI) [14] was not observed. However, the difference between test measurements was small, and mammography's moderate heterogeneity, wider LOA, and tendency to "miss" smaller tumors $(\leq 2.0 \mathrm{~cm})$ indicate greater variability for agreement with pathology. Consequently, combining MRI and mammography did not improve tumor measurement compared with MRI alone. In addition, a tendency for large mammographic measurements in the presence of pCR suggests that mammography may lead to overly radical surgery when $\mathrm{pCR}$ is achieved. Mammographic tumor measurements were frequently not possible due to breast density, reflected in the younger age of those women [49]. These findings therefore suggest that MRI would be the preferred test in this setting.

Direct comparison of MRI and clinical examination showed no systematic bias in MRI's measurement of residual tumor; relatively large underestimation, moderate heterogeneity and wider LOA for clinical examination were observed, suggesting greater variability for agreement with pathology. In addition, apart from one case, tumors with pathologic measurements of $>2.0 \mathrm{~cm}$ were "missed" only by clinical examination, highlighting the potential for inadequate resection if surgical planning was based on clinical examination alone. While better overall agreement between MRI and pathology suggest that MRI is the more appropriate assessment method, it is possible that a combination of US and clinical examination may be superior to either test individually [50], but that testing strategy could not be explored in this analysis. The relative performance of test combinations should be considered in future studies.

Data from single studies have suggested that underestimation by MRI is common in HER2- patients [16] or those treated with taxane-containing regimens [17], but 
previous study-level meta-analyses were unable to further explore the effect of these variables. Similar effects were not observed in our IPD analysis. For patients with data available on HER2 status, MRI performed comparably regardless of tumor biology. Although that analysis was based on relatively few studies, the combined sample size is substantially larger than the previous study exploring the effect of this variable, and the studies that did not contribute data predate the routine testing of HER2. Furthermore, contrary to previous reports, a slight bias towards underestimation (and poorer overall agreement with pathology) was found in patients treated with non-taxane-based NAC. However, although more detailed analyses were attempted, statistical models were unstable and therefore the results presented are primarily descriptive. Further exploration of the effect of these characteristics on measurement accuracy is warranted in large primary studies, controlling for the effect other potentially important covariates.

Given that not all eligible studies contributed IPD to this meta-analysis, selection bias may have been introduced. Although studies in this analysis were similar in most respects to the broader population of eligible studies [14], a higher proportion of T3 tumors and stage III disease was apparent. Other differences suggest that included studies are more applicable to current practice (i.e. NAC with taxanes was more common), and less susceptible to changes in tumor dimensions between MRI and pathologic measurement (i.e. shorter interval between tests). Our IPD analysis also included a larger number of studies than the only previous (study-level) meta-analysis utilising appropriate statistical techniques to address this clinical question [14] (see Additional file 1: Appendix 1).

Although MDs and LOA are the most methodologically appropriate measures of agreement between MRI and pathology [15], there was no clear indication to consider either absolute or relative differences between the tests in our analysis. Plots of the data suggest that the absolute MDs reported here are likely to be most applicable to mid-sized tumors, but may differ for small or large residual cancers. However, analyses of absolute and relative differences were comparable, and therefore inferences about MRI and its performance compared to alternative tests are likely to be robust.

Due to pCR being achieved in a minority of patients (between $7.1 \%$ and $27.5 \%$ in the included studies), analyses of measurement errors in the presence of $\mathrm{pCR}$ are based on relatively small sample sizes and should therefore be interpreted cautiously. Furthermore, to standardise the definition of $\mathrm{pCR}$ across studies, this analysis considered the presence of invasive cancer only. This represents an advance in methods over previous analyses by reducing the potential for heterogeneity and improving the clinical applicability of pooled estimates.
However, tests may differ in their ability to visualise DCIS or calcifications [11], and hence the accuracy of MRI and alternative tests to measure those outcomes may differ from our estimates. Our findings that alternative tests could not evaluate residual tumor in a proportion of patients should also be interpreted with awareness that corresponding data about non-evaluable tumors by MRI were unavailable.

\section{Conclusion}

Our meta-analysis is the largest and most statistically appropriate evaluation of the agreement between MRI and pathologic residual tumor size post-NAC, and the only meta-analysis on this topic using IPD methodology. Our work suggests that there is no systematic bias in MRI's measurement of residual invasive tumor, but that both over- and underestimation by MRI is possible, with LOA large enough to be of clinical importance. MRI's performance was generally superior to that of US, mammography, and clinical examination, and in light of those findings, MRI may be considered the most appropriate test in this setting. However, large MRI measurements are possible in a small proportion of $\mathrm{pCR}$ cases, and patient characteristics that render tumors non-evaluable by US may contribute to inaccurate size measurements by MRI; those potential disadvantages should be considered in the choice of test. Furthermore, it is possible that a combination of US and clinical examination may be superior to those tests individually, and such a testing strategy has potential advantages over MRI in terms of lower cost and greater accessibility. Combinations of alternative tests, and their performance relative to MRI, should be explored in future studies.

\section{Additional file}

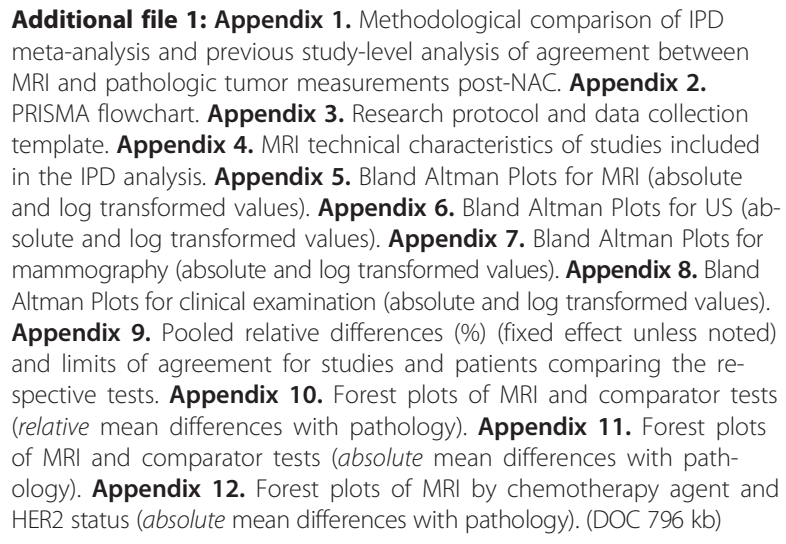

Competing interests

SCP receives research funding from Philips Healthcare. The other authors declare no competing interests. 


\section{Authors' contributions}

MLM conceived and co-ordinated the study, conducted the literature searches and review of studies, performed the statistical analysis, and drafted the manuscript. PM conceived the statistical methods used, advised on data analysis and interpretation, and contributed to drafting the manuscript. LI advised on methodological aspects, data interpretation and contributed to drafting the manuscript. FS advised on MRI technical issues and clinical aspects, and contributed to drafting the manuscript. EPM advised on clinical aspects and contributed to drafting the manuscript. GvM advised on clinical aspects and contributed to drafting the manuscript. VG collected and assembled data and contributed to drafting the manuscript. SCP collected and assembled data and contributed to drafting the manuscript. FCW collected and assembled data and contributed to drafting the manuscript. JHC collected and assembled data and contributed to drafting the manuscript. MB collected and assembled data and contributed to drafting the manuscript. LM collected and assembled data and contributed to drafting the manuscript. EY collected and assembled data and contributed to drafting the manuscript. VL collected and assembled data and contributed to drafting the manuscript. $\mathrm{NH}$ conceived the study, advised on literature searches and study eligibility, advised on clinical aspects and data interpretation, and contributed to drafting the manuscript. All authors read and approved the final manuscript.

\section{Acknowledgements}

This work was partly funded by National Health and Medical Research Council (NHMRC Australia) program grant 633003 to the Screening \& Test Evaluation Program. M. L. Marinovich was supported by a NHMRC postgraduate scholarship. N. Houssami receives research support through a National Breast Cancer Foundation (NBCF Australia) Practitioner Fellowship. The funding bodies had no role in the study design; in the collection, analysis, and interpretation of data; in the writing of the manuscript; and in the decision to submit the manuscript for publication.

\section{Author details}

${ }^{1}$ Screening and Test Evaluation Program (STEP), Sydney School of Public Health, The University of Sydney, A27, Edward Ford Building, Sydney, NSW 2006, Australia. ²Dipartimento di Scienze Biomediche per la Salute, Università degli Studi di Milano, Unità di Radiologia, IRCCS Policlinico San Donato, Piazza E. Malan 2, San Donato Milanese, Milano, Italy. ${ }^{3} \mathrm{MD}$ Anderson Cancer Center Orlando, 1400 South Orange Avenue, MP 700, Orlando, FL 32806, USA. ${ }^{4}$ German Breast Group \& Universitäts-Frauenklinik Frankfurt, Martin-Behaim-Str. 12, 63263 Neu-Isenburg, Germany. ${ }^{5}$ University of Padova, Division of Medical Oncology 2, Istituto Oncologico Veneto IRCCS, Padova, Italy. ${ }^{6}$ Department of Radiology, University of Washington, 825 Eastlake Ave E, G3-200, Seattle, WA 98109-1023, USA. 'Division of General Surgery, Sunnybrook Health Sciences Centre, 2075 Bayview Avenue, Toronto, ON M4C 5T2, Canada. ${ }^{8}$ School of Medicine, Jeju National University Hospital, Aran 13gil 15(ara-1 dong), Jeju-si, Jeju-do, South Korea. ${ }^{9}$ Berkshire Cancer Centre, Royal Berkshire NHS Foundation Trust, London Road, Reading RG1 5AN, UK. ${ }^{10}$ Direzione Radiodiagnostica, Fondazione del Piemonte per I'Oncologia-IRCCS, Str. Prov.142, Candiolo, Torino, Italy. ${ }^{11}$ Department of Radiology, Brigham and Women's Hospital, 75 Francis St, Boston, MA 02115, USA. ${ }^{12}$ Institute of Radiology, University of Udine, p.le S.M. della Misericordia, 15, 33100 Udine, Italy.

Received: 24 February 2015 Accepted: 29 September 2015 Published online: 08 October 2015

\section{References}

1. Marinovich ML, Houssami N, Macaskill P, Sardanelli F, Irwig L, Mamounas EP, et al. Meta-analysis of Magnetic Resonance Imaging in Detecting Residual Breast Cancer After Neoadjuvant Therapy. J Natl Cancer Inst. 2013;105:321-33.

2. Eisenhauer EA, Therasse P, Bogaerts J, Schwartz LH, Sargent D, Ford R, et al. New response evaluation criteria in solid tumours: Revised RECIST guideline (version 1.1). Eur J Cancer. 2009;45:228-47.

3. Irwig L, Bennetts A. Quality of life after breast conservation or mastectomy: A systematic review. Aust New Zealand J Surg. 1997;67:750-4.

4. Delille JP, Slanetz PJ, Yeh ED, Halpern EF, Kopans DB, Garrido L. Invasive ductal breast carcinoma response to neoadjuvant chemotherapy: Noninvasive monitoring with functional MR imaging - Pilot study. Radiology. 2003;228:63-9.
5. Genson CC, Blane CE, Helvie MA, Waits SA, Chenevert TL. Effects on breast MRI of artifacts caused by metallic tissue marker clips. Am J Roentgenol. 2007;188:372-6.

6. Kolb TM, Lichy J, Newhouse JH. Comparison of the Performance of Screening Mammography, Physical Examination, and Breast US and Evaluation of Factors that Influence Them: An Analysis of 27,825 Patient Evaluations. Radiology. 2002;225:165-75.

7. Sardanelli F, Giuseppetti GM, Panizza P, Bazzocchi M, Fausto A, Simonetti G, et al. Sensitivity of MRI versus mammography for detecting foci of multifocal, multicentric breast cancer in fatty and dense breasts using the whole-breast pathologic examination as a gold standard. Am J Roentgenol. 2004;183:1149-57.

8. Bosch AM, Kessels AGH, Beets GL, Rupa JD, Koster D, Van Engelshoven JMA, et al. Preoperative estimation of the pathological breast tumour size by physical examination, mammography and ultrasound: A prospective study on 105 invasive tumours. Eur J Radiol. 2003:48:285-92.

9. Baker JA, Soo MS, Rosen EL. Artifacts and Pitfalls in Sonographic Imaging of the Breast. Am J Roentgenol. 2001;176:1261-6.

10. Hieken TJ, Harrison J, Herreros J, Velasco JM. Correlating sonography, mammography, and pathology in the assessment of breast cancer size. Am J Surg. 2001;182:351-4.

11. Lehman CD. Magnetic Resonance Imaging in the Evaluation of Ductal Carcinoma In Situ. JNCI Monographs. 2010;41:150-1.

12. Tucker FL. Imaging-assisted large-format breast pathology: Program rationale and development in a nonprofit health system in the United States. Int J Breast Cancer 2012;1. http://www.hindawi.com/journals/ijbc/ 2012/171792/cta/

13. Yeh E, Slanetz P, Kopans DB, Rafferty E, Georgian-Smith D, Moy L, et al. Prospective comparison of mammography, sonography, and MRI in patients undergoing neoadjuvant chemotherapy for palpable breast cancer. Am J Roentgenol. 2005;184:868-77.

14. Marinovich ML, Macaskill P, Irwig L, Sardanelli F, Von Minckwitz G,

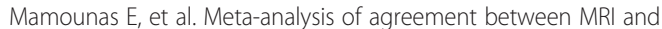
pathologic breast tumour size after neoadjuvant chemotherapy. Br J Cancer. 2013;109:1528-36.

15. Bland JM, Altman DG. Statistical methods for assessing agreement between two methods of clinical measurement. Lancet. 1986:327:307-10.

16. Chen JH, Feig B, Agrawal G, Yu H, Carpenter PM, Mehta RS, et al. MRI evaluation of pathologically complete response and residual tumors in breast cancer after neoadjuvant chemotherapy. Cancer. 2008;112:17-26.

17. Denis F, Desbiez-Bourcier AV, Chapiron C, Arbion F, Body G, Brunereau L. Contrast enhanced magnetic resonance imaging underestimates residual disease following neoadjuvant docetaxel based chemotherapy for breast cancer. Eur J Surg Oncol. 2004;30:1069-76.

18. Bossuyt PM, Irwig L, Craig J, Glasziou P. Comparative accuracy: assessing new tests against existing diagnostic pathways. Br Med J. 2006:332:1089-92.

19. Marinovich ML, Sardanelli F, Ciatto S, Mamounas E, Brennan M, Macaskill P, et al. Early prediction of pathologic response to neoadjuvant therapy in breast cancer: Systematic review of the accuracy of MRI. Breast. 2012;21:669-77.

20. Ogston KN, Miller ID, Payne S, Hutcheon AW, Sarkar TK, Smith I, et al. A new histological grading system to assess response of breast cancers to primary chemotherapy: Prognostic significance and survival. Breast. 2003;12:320-7.

21. Higgins JPT, Thompson SG, Deeks JJ, Altman DG. Measuring inconsistency in meta-analyses. Br Med J. 2003;327:557-60.

22. Woodward M. Basic analytical procedures. Epidemiology: Study design and data analysis. Boca Raton: Chapman \& Hall/CRC; 1999. p. 31-105.

23. Bland M. Significance tests. In: Bland M, editor. An Introduction to Medical Statistics. 3rd ed. Oxford: Oxford University Press; 2000. p. 137-55.

24. Wright FC, Zubovits J, Gardner S, Fitzgerald B, Clemons M, Quan ML, et al. Optimal assessment of residual disease after neo-adjuvant therapy for locally advanced and inflammatory breast cancer - Clinical examination, mammography, or magnetic resonance imaging? J Surg Oncol. 2010;101:604-10.

25. Partridge SC, Gibbs JE, Lu Y, Esserman LJ, Sudilovsky D, Hylton NM. Accuracy of MR imaging for revealing residual breast cancer in patients who have undergone neoadjuvant chemotherapy. Am J Roentgenol. 2002;179:1193-9.

26. Prati R, Minami CA, Gornbein JA, Debruhl N, Chung D, Chang HR. Accuracy of clinical evaluation of locally advanced breast cancer in patients receiving neoadjuvant chemotherapy. Cancer. 2009;115:1194-202.

27. Segara D, Krop IE, Garber JE, Winer E, Harris L, Bellon JR, et al. Does MRI predict pathologic tumor response in women with breast cancer undergoing preoperative chemotherapy? J Surg Oncol. 2007;96:474-80. 
28. Akazawa K, Tamaki Y, Taguchi T, Tanji Y, Miyoshi Y, Kim SJ, et al. Preoperative evaluation of residual tumor extent by three-dimensional magnetic resonance imaging in breast cancer patients treated with neoadjuvant chemotherapy. Breast J. 2006;12:130-7.

29. Guarneri V, Pecchi A, Piacentini F, Barbieri E, Dieci MV, Ficarra G, et al. Magnetic resonance imaging and ultrasonography in predicting infiltrating residual disease after preoperative chemotherapy in stage II-III breast cancer. Ann Surg Oncol. 2011:18:2150-7.

30. Rosen EL, Blackwell KL, Baker JA, Soo MS, Bentley RC, Yu D, et al. Accuracy of MRI in the Detection of Residual Breast Cancer After Neoadjuvant Chemotherapy. Am J Roentgenol. 2003;181:1275-82.

31. Nakahara H, Yasuda Y, Machida E, Maeda Y, Furusawa H, Komaki K, et al. MR and US imaging for breast cancer patients who underwent conservation surgery after neoadjuvant chemotherapy: comparison of triple negative breast cancer and other intrinsic subtypes. Breast Cancer. 2011;18:152-60.

32. Julius T, Kemp SEG, Kneeshaw PJ, Chaturvedi A, Drew PJ, Turnbull LW. MRI and conservative treatment of locally advanced breast cancer. Eur J Surg Oncol. 2005;31:1129-34.

33. Balu-Maestro C, Chapellier C, Bleuse A, Chanalet I, Chauvel C, Largillier R. Imaging in evaluation of response to neoadjuvant breast cancer treatment benefits of MRI. Breast Cancer Res Tr. 2002;72:145-52.

34. Bhattacharyya M, Ryan D, Carpenter R, Vinnicombe S, Gallagher CJ. Using MRI to plan breast-conserving surgery following neoadjuvant chemotherapy for early breast cancer. Br J Cancer. 2008;98:289-93.

35. Bollet MA, Thibault F, Bouillon K, Meunier M, Sigal-Zafrani B, Savignoni A, et al. Role of Dynamic Magnetic Resonance Imaging in the Evaluation of Tumor Response to Preoperative Concurrent Radiochemotherapy for Large Breast Cancers: A Prospective Phase II Study. Int J Radiat Oncol Biol Phys. 2007:69:13-8.

36. Montemurro F, Martincich L, De Rosa G, Cirillo S, Marra V, Biglia N, et al. Dynamic contrast-enhanced MRI and sonography in patients receiving primary chemotherapy for breast cancer. Eur Radiol. 2005;15:1224-33.

37. Chen X, Moore MO, Lehman CD, Mankoff DA, Lawton TJ, Peacock S, et al. Combined use of MRI and PET to monitor response and assess residual disease for locally advanced breast cancer treated with neoadjuvant chemotherapy. Acad Radiol. 2004;11:1115-24.

38. Londero V, Bazzocchi M, Del Frate C, Puglisi F, Di Loreto C, Francescutti G, et al. Locally advanced breast cancer: Comparison of mammography, sonography and MR imaging in evaluation of residual disease in women receiving neoadjuvant chemotherapy. Eur Radiol. 2004;14:1371-9.

39. Bodini M, Berruti A, Bottini A, Allevi G, Fiorentino C, Brizzi MP, et al. Magnetic resonance imaging in comparison to clinical palpation in assessing the response of breast cancer to epirubicin primary chemotherapy. Breast Cancer Res Tr. 2004;85:211-8.

40. Weatherall PT, Evans GF, Metzger GJ, Saborrian MH, Leitch AM. MRI vs. histologic measurement of breast cancer following chemotherapy: Comparison with x-ray mammography and palpation. J Magn Reson Im. 2001;13:868-75.

41. Moon HG, Han W, Lee JW, Ko E, Kim EK, Yu JH, et al. Age and HER2 expression status affect MRI accuracy in predicting residual tumor extent after neo-adjuvant systemic treatment. Ann Oncol. 2009;20:636-41.

42. Dose-Schwarz J, Tiling R, Avril-Sassen S, Mahner S, Lebeau A, Weber C, et al. Assessment of residual tumour by FDG-PET: Conventional imaging and clinical examination following primary chemotherapy of large and locally advanced breast cancer. Br J Cancer. 2010;102:35-41.

43. Kim HJ, Im YH, Han BK, Choi N, Lee J, Kim JH, et al. Accuracy of MRI for estimating residual tumor size after neoadjuvant chemotherapy in locally advanced breast cancer: Relation to response patterns on MRI. Acta Oncol. 2007:46:996-1003.

44. Martincich L, Montemurro F, De Rosa G, Marra V, Ponzone R, Cirillo S, et al. Monitoring response to primary chemotherapy in breast cancer using dynamic contrast-enhanced magnetic resonance imaging. Breast Cancer Res Tr. 2004;83:67-76.

45. Esserman L, Kaplan E, Partridge S, Tripathy D, Rugo H, Park J, et al. MR phenotype is associated with response to doxorubicin and cyclophosphamide neoadjuvant chemotherapy in stage III breast cancer Ann Surg Oncol. 2001;8:549-59.

46. Choi JH, Lim HI, Lee SK, Kim WW, Kim SM, Cho E, et al. The role of PET CT to evaluate the response to neoadjuvant chemotherapy in advanced breast cancer: Comparison with ultrasonography and magnetic resonance imaging. J Surg Oncol. 2010;102:392-7.
47. Montagna E, Bagnardi V, Rotmensz N, Viale G, Pruneri G, Veronesi P, et al. Pathological complete response after preoperative systemic therapy and outcome: Relevance of clinical and biologic baseline features. Breast Cancer Res Tr. 2010;124:689-99.

48. Bear HD, Anderson S, Smith RE, Geyer J, Mamounas EP, Fisher B, et al. Sequential preoperative or postoperative docetaxel added to preoperative doxorubicin plus cyclophosphamide for operable breast cancer: National surgical adjuvant breast and bowel project protocol B-27. J Clin Oncol. 2006;24:2019-27

49. Checka CM, Chun JE, Schnabel FR, Lee J, Toth $\mathrm{H}$. The relationship of mammographic density and age: Implications for breast cancer screening. Am J Roentgenol. 2012;198:W292-5.

50. Herrada J, lyer RB, Atkinson EN, Sneige N, Buzdar AU, Hortobagyi GN. Relative value of physical examination, mammography, and breast sonography in evaluating the size of the primary tumor and regional lymph node metastases in women receiving neoadjuvant chemotherapy for locally advanced breast carcinoma. Clin Cancer Res. 1997;3:1565-9.

\section{Submit your next manuscript to BioMed Central and take full advantage of:}

- Convenient online submission

- Thorough peer review

- No space constraints or color figure charges

- Immediate publication on acceptance

- Inclusion in PubMed, CAS, Scopus and Google Scholar

- Research which is freely available for redistribution 